\section{Histopathology of the synovial tissue: perspectives for biomarker development in chronic inflammatory arthritides}

\author{
A. Manzo, S. Bugatti, R. Caporali, C. Montecucco \\ Rheumatology and Translational Immunology Research Laboratories (LaRIT), Division of Rheumatology, \\ IRCCS Policlinico San Matteo Foundation/University of Pavia, Italy
}

\section{SUMMARY}

The histopathological and molecular analysis of the synovial tissue has contributed to fundamental advances in our comprehension of arthritis pathogenesis and of the mechanisms of action of currently available treatments.

On the other hand, its exploitation in clinical practice for diagnostic or prognostic purposes as well as for the prediction of treatment response to specific disease-modifying anti-rheumatic drugs is still limited. In this review, we present an overview of recent advances in the field of synovial tissue research with specific reference to the methods for synovial tissue collection, approaches to synovial tissue analysis and current perspectives for the exploitation of synovial tissue-derived biomarkers in chronic inflammatory arthritides.

Key words: Synovitis; Arthritis; Histopathology.

\section{INTRODUCTION}

$\mathrm{D}$ ata derived from the histopathological analysis of target tissues are an integral component of current classification criteria or routine diagnostic/prognostic work-up in several diseases, including cancers and immune-inflammatory conditions. Relevant examples of the latter include, but are not limited to, the analysis of salivary glands in Sjögren's syndrome (1), the kidney in systemic lupus erythematosus (2) and the gut mucosa in inflammatory-bowel diseases (3).

This is only partly the case with regard to synovial tissue analysis, which still offers a relatively limited application in routine clinical practice. Although microbiological, molecular or histological assessment of the synovial tissue can assist clinicians in the diagnosis of joint infections, musculoskeletal manifestations in course of rare diseases (i.e. sarcoidosis, amyloidosis, haemochromatosis, ochronosis, multicentric reticulohistiocytosis) and synovial tu- mours (4), synovial pathology is still not integrated in standard-of-care management of most common forms of inflammatory arthropathies. No synovial-derived markers are actually exploited in clinical contexts to predict disease onset, diagnosis, clinical outcomes, drug toxicity or to guide personalized treatment choices in rheumatoid arthritis (RA), psoriatic arthritis (PsA) and other forms of spondyloarthritis (SpA). Thus, beside the established role of synovial tissue analysis for pathobiological research (5-8), as a source of surrogate endpoints for drug development (9) and to elucidate the mode of action of treatments (10-12), its potential to improve the management of arthritis in real life settings remains the object of research (13).

In the following sections, we present a critical summary of the main studies that have focused on this goal and that have contributed to strengthening the scientific rationale of this perspective. We have clustered published data according to specific phases of arthritis evolution, with primary
Corresponding author: Antonio Manzo

Rheumatology and Translational Immunology Research

Laboratories (LaRIT),

Division of Rheumatology, IRCCS Policlinico San Matteo

Foundation/University of Pavia

P.le Golgi, 19 - 27100 Pavia, Italy

E-mail: antonio.manzo@unipv.it 
emphasis on three of those in which validation of novel biomarkers would be relevant in current clinical practice:

1) early undifferentiated arthritis,

2) arthritis refractory to first line treatments,

3 ) arthritis in remission.

\section{SYNOVIAL PATHOTYPES}

The synovium is a specialized connective tissue lining the inner surface of diarthrodial joints capsules, tendon sheaths and bursae. Due to its intrinsic deformability, porosity and through the secretory activity of its cellular components, it plays a fundamental role in physiological joint movement, synovial fluid homeostasis, cartilage lubrication and chondrocyte nutrition. These functions are supported by its con- nection with independent anatomic environments:

1) the joint cavity, through a superficial lining layer of fibroblast- and macrophage-like cells lacking tight junctions and devoid of a basement membrane;

2) the systemic circulation, through small blood vessels and lymphatics, controlling cell trafficking, plasma ultrafiltration and fluid clearance;

3) the central nervous system, through sympathetic and sensory nerve fibres regulating vascular tone and nociception.

Among these structures, a few scattered adipocytes, macrophages, quiescent/low proliferating fibroblasts (14) and multipotent mesenchymal stem cells (15) complete the synovial cell architecture in normal conditions.

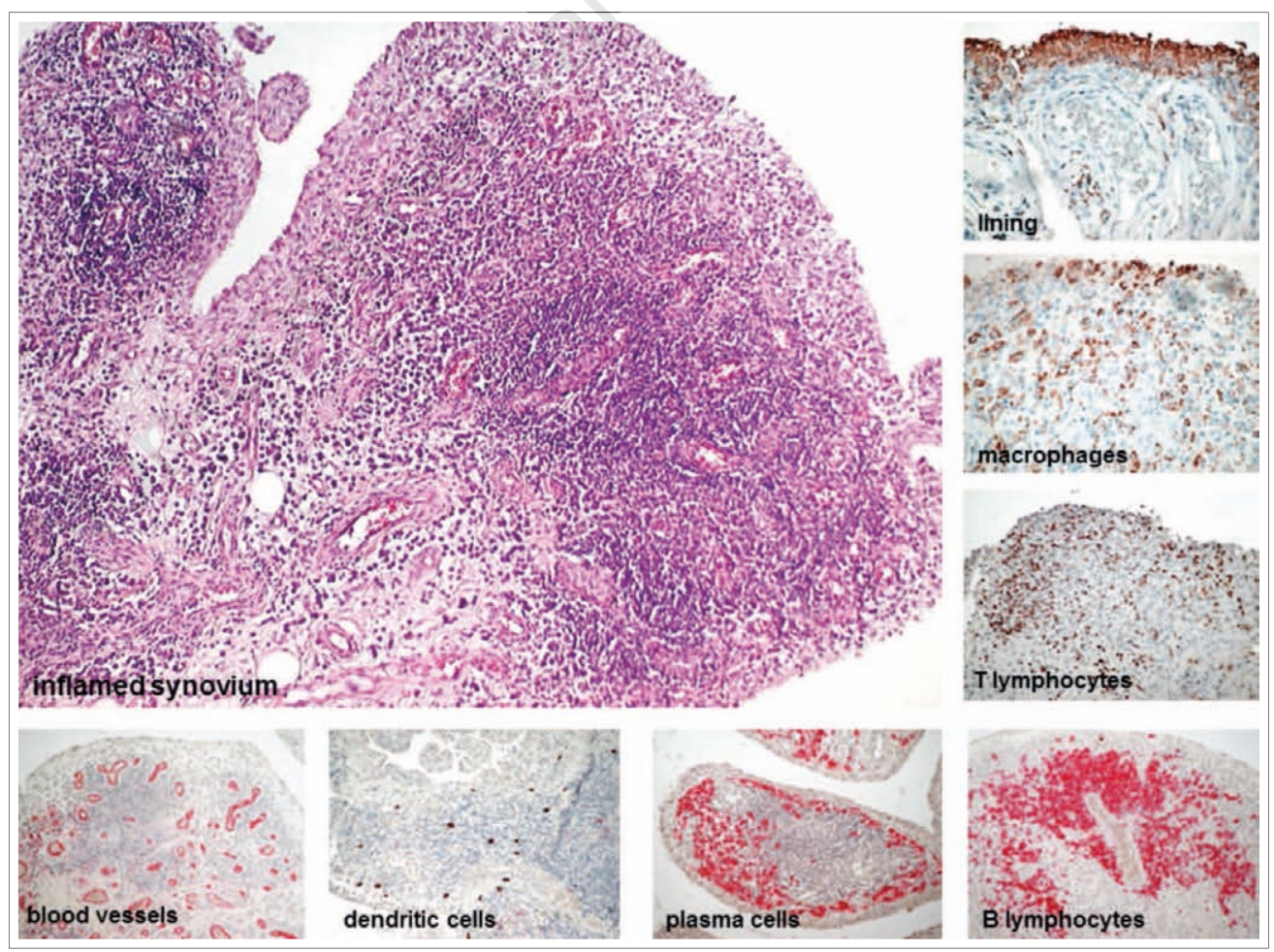

Figure 1 - The inflamed synovium in course of arthritis. The general histopathological appearance of the inflamed synovium (large panel, hematoxylin and eosin) in course of arthritis and of specific cellular components (small panels, immunostainings) are shown. Inflammatory features include hyperplasia of the lining layer, infiltration of the synovial sublining by myeloid and lymphoid cells including macrophages (CD68 staining, brown), T lymphocytes (CD3, brown), B lymphocytes (CD20, red), plasma cells (CD138, red), dendritic cells (CD83, brown), and new blood vessel formation (neoangiogenesis) (CD31, red). 

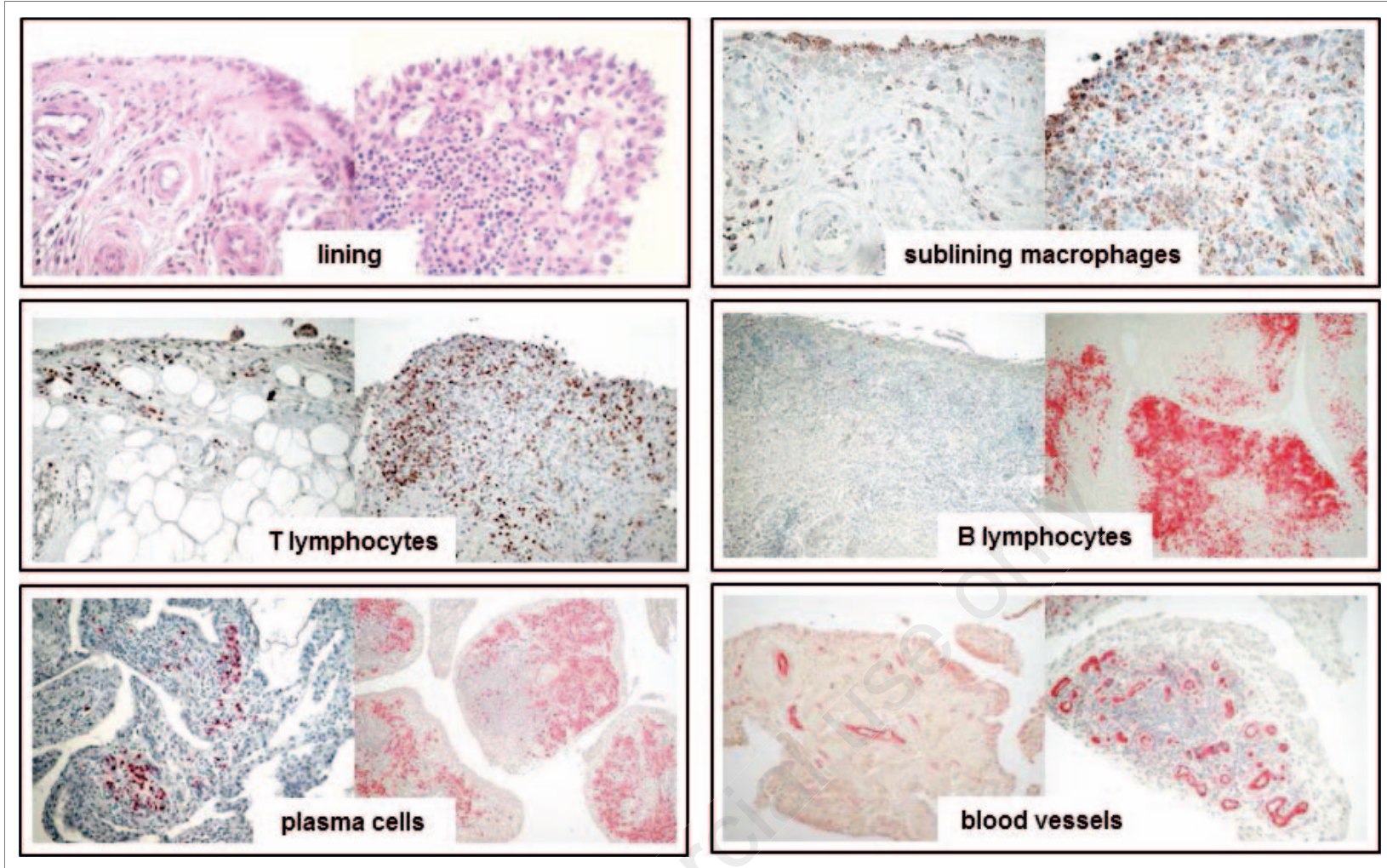

Figure 2 - Synovial tissue heterogeneity. Inter-individual variations of synovial inflammatory features in course of arthritis are shown. High quantitative variability is observed for the degree of lining layer thickness, of sublining infiltration by myeloid and lymphoid cells such as macrophages (CD68 staining, brown), T lymphocytes (CD3, brown), B lymphocytes (CD20, red), plasma cells (CD183, red), and of new blood vessel formation (CD31, red).

The inflammatory process taking place in course of RA or SpA leads to detectable pathobiological modifications virtually related to all the above-mentioned components (Figure 1). These can include dysregulated angiogenesis (16), local recruitment and organization of myeloid and lymphoid cells $(5,17)$, enhanced stromal cell proliferation and epigenetic changes $(14,18-20)$, changes induced by local haematopoietic-stromal cell crosstalks (21-23), lymphocyte activation and differentiation (19, 24-27).

Supporting the potential utility of the assessment of synovitis for clinical stratification, these processes are not uniformly and synchronously captured in the inflamed joint, but are characterized by a degree of inter-individual heterogeneity (Figure 2). This variability is determined by the gradients that delineate the expression level of most inflammatory markers and by the possible unrelated expression of different markers within single tissues $(28,29)$. Strengthening the pathobiological rationale of this picture, when synovitis is approached multi-parametrically, it is actually possible to distinguish gross pathological categories that rely on clustered and congruent histopathological and molecular features. This general concept, previously proposed in independent studies (30-33), has been recently refined through the delineation of four primary patterns of synovitis (34):

1) the lymphoid phenotype, characterized by enrichment of genes related to $\mathrm{B} / \mathrm{T}$ lymphocyte activation and differentiation, cytokine signalling including the Jak/STAT pathway and IL-17 signalling;

2) the myeloid phenotype, also characterized by processes associated with 
chemotaxis, TNF $\alpha$ and IL- $1 \beta$ production, Toll-like receptor signalling, $\mathrm{Fc} \gamma$ receptor meditated phagocytosis;

3) the fibroid phenotype, enriched for genes associated with TGF $\beta$ and BMP signalling, but lacking enrichment of any immune system processes;

4) the low inflammatory phenotype, showing only enrichment for inflammatory response and wound response processes.

It is worth mentioning that, if on the one hand these studies provide a solid biologic substrate for synovial analyses and for clinical translation, on the other hand they leave unsolved some critical issues. In particular, since synovial parameters are influenced by disease activity (35), whether these patterns reflect dynamic phases of the inflammatory process or stable pathological characteristics of specific RA/SpA subsets remains to be defined.

\section{SYNOVIAL TISSUE BIOPSY}

In addition to the inputs derived from basic comprehension of synovial pathobiology, the opportunities to exploit the synovial membrane for biomarker discovery have been considerably widened in recent years by the development of mini-invasive percutaneous approaches allowing tissue retrieval across progressive phases of the disease. These contemplate blind needle biopsy, arthroscopic biopsy and ultrasound (US)-guided biopsy (the latter including a quick core needle and a portal and forceps technical variants). All these techniques have been shown to allow large and small joint sampling (36-38), to be applicable from early stages of the disease (39-41), to be well tolerated $(38,42,43)$ and to allow collection of good quality tissue for histopathological analyses $(44,45)$. Despite parallel comparative analyses are still ongoing, arthroscopic and US-guided biopsies are currently favoured in clinical research due to the opportunity of visualizing the synovium during the procedure, thus increasing sampling specificity, applicability in quiescent joints and repeatability after treatment.
The above technical advancements, directed towards improving accessibility to synovial tissue, have been integrated with studies focused on the approach to synovial tissue sampling. In particular, since synovial analysis relies on retrieval of only a few specimens from a single joint, a fundamental issue related to its clinical applicability to systemic diseases is whether the synovial membrane in one joint is actually representative of that seen in other joints. In this perspective, Kraan et al. (36) analyzed the characteristics of the synovium in paired arthroscopic samples of the knee and small joints (wrists or metacarpophalangeal joints) in 9 patients with RA. Despite marked variation among patients for each marker, no differences were detected in the distribution of fibroblast-like synoviocytes, macrophages, $\mathrm{T}$ cells and plasma cells in different anatomic sites. In addition, significant correlations were reported in all sublining parameters between knee and small joints, supporting the view that synovial sublining infiltration in one joint can be representative of the systemic process in RA.

Due to the intra-tissue heterogeneity of synovial inflammation, a complementary issue that has been the object of intense research relates to the amount of tissue required to achieve a representative analysis of the inflammatory status in each joint. Supporting the relevance of analysing multiple areas of the joint (rather than multiple sections from a single area), Dolhain et al. (46), through the immunohistological assessment of synovial samples from large joints, demonstrated that a variance of less than $10 \%$ for $\mathrm{T}$ cell markers and expression of activation antigens can be reached when at least six different specimens are examined. Accordingly, four to seven random synovial fragments have been shown to allow detection of two-fold change in gene expression with $25 \%$ sampling error by quantitative PCR (47). More recent studies focused on small joints, in which synovial volume is much reduced, confirmed the requirement of a minimum of four independent samples for reliable histopathological analysis, a target that can be achieved by US-guided needle 
biopsy in approximately $80 \%$ of the joints with grade 3 synovial thickness (48).

Taken together, these studies suggest that representative data can be obtained when a limited number of biopsy samples from different areas of one joint are investigated in RA, supporting the reliability and feasibility of the procedure. Additional work is required to generalize further these conclusions to different diseases, techniques and markers.

\section{EARLY UNDIFFERENTIATED ARTHRITIS: DEVELOPMENT OF DIAGNOSTIC BIOMARKERS}

A wide discovery platform for the development of biomarkers and prediction models, still characterized by considerable unmet needs, is the early arthritis phase. Since early diagnosis and treatment are linked to better long-term outcomes $(49,50)$, the development of novel tools able to improve the diagnostic performance in patients with recent onset joint swelling is actually seen as a relevant target with a direct impact on current clinical practice.

Several synovial histopathological studies in the past have contributed to advance current knowledge in this direction, either indirectly through comparative analyses of differentially expressed markers in established diseases, or directly through the longitudinal evaluation in undifferentiated arthritis patients.

Proof-of-concept evidence supporting the possibility to track disease-associated changes in situ derives from independent cross-sectional studies investigating the pattern of knee synovitis in RA and SpA. Examples of histological parameters shown to be differently expressed are vascularity $(51,52)$, angiopoietin- $2(53,54)$ and CD163+ macrophages $(52,55)$, all shown to be significantly increased in SpA vs RA synovium; lining layer thickness $(51,52)$ and the MHC-human cartilage gp39 complex $(52,56)$, augmented in or restricted to RA. The existence of differences has been strengthened further through comparative analysis by pan-genomic microarrays (57). Despite these data, however, only a limited number of studies have analysed the actual diagnostic value of synovial histopathology in longitudinal cohorts of patients with early undifferentiated arthritis. One of the largest data-sets available to date refers to the study performed by Kraan et al. (41), who evaluated 95 patients with recent onset $(<1$ year) and unclassified arthritis, across a follow-up of at least 2 years after biopsy. Comparison of baseline synovial characteristics of the RA patients $v s$ the pooled non-RA patients at the end of follow-up by logistic regression identified CD38+ plasma cells and CD22+ B cells as independent predictors and best discriminating markers, suggesting that the histopathological analysis of knee synovium in patients with early arthritis can be used to differentiate RA from other conditions. No evidence for a discriminative value of sub-lining cell aggregates (dichotomously stratified as present/absent) was obtained, instead, in another study based on a similar experimental setting through the prospective analysis of 39 undifferentiated arthritis patients (39).

While these data suggest a potential role of specific synovial markers for early diagnostic discrimination, the most likely scenario for clinical exploitation could be their integration into multivariable prediction models (not necessarily restricted to synovial parameters). This concept has been challenged by Baeten et al. (58) who analysed, in 53 patients with unclassified inflammatory arthritis, the diagnostic predictive value of single histological markers (developed from a learning cohort) combined in a multiparameter decision tree. Diagnosis, evaluated after a follow up of 6 months, was comprehensive of RA, SpA and other diseases (gout, chondrocalcinosis, villonodular synovitis, osteoarthritis, multiple myeloma, Sudeck syndrome and polymyalgia rheumatica). Despite the wide clinical spectrum, the developed algorithm (based on the assessment of synovial crystal depositions, anti-citrulline staining, staining for the MHC-human cartilage gp39 complex, microscopic vascularity, lining layer thickness and macroscopic vascular pattern) showed an overall positive predic- 
tive value of $81 \%$ and higher performance compared to a similar multiparameter algorithm exclusively based on classic (clinical, serological, genetic and radiographic) diagnostic features.

Further multi-centric prospective studies in large patient cohorts and through standardized assessment protocols might contribute to promoting translation of these promising data into clinical practice.

\section{DISEASE STRATIFICATION: DEVELOPMENT OF PREDICTIVE BIOMARKERS OF RESPONSE TO TREATMENT}

Beyond the development of diagnostic biomarkers, another relevant target of arthritis research is the development of new tools able to predict response to individual therapies. The rationale of this goal is based on the heterogeneity that characterizes chronic inflammatory arthritides, both in their natural evolution as well as in the response to specific drugs. If on the one hand patients affected by the same disease can show a heterogeneous response to the same molecule, on the other, the same molecule can induce a similar response in patients affected by different forms of arthritis. This observation has led to conceive the perspective of a parallel taxonomy of chronic inflammatory arthritides firmly rooted in shared pathogenetic mechanisms and reflected in shared response to treatments.

In this context, the specific opportunities offered by synovial tissue analysis in terms of dissecting molecular aspects of disease (compared to clinical or imaging approaches), clearly represent a fundamental resource. Although this concept may be applicable to any treatment, biologic therapies have represented a primary research platform in recent years, due to unmet clinical needs (overall ACR50 response rate in RA is less than 50\%) (59), high financial costs and their intrinsic pharmacological characteristics based on interactions with known molecular targets.

In the next paragraphs, a selection of most relevant studies published so far, primarily focused on synovial predictors of response to anti-TNF and Rituximab in RA, is discussed.

\section{Predictors of response to anti-TNF}

Initial proof-of-concept evidence suggesting the possibility to capture predictive information based on synovial assessments has been provided by van der Pouw Kraan et al. (60) who, through large-scale gene expression profiling by microarrays and pathway analysis in 18 RA patients starting treatment with infliximab, observed a positive association between pre-treatment synovial inflammatory activity and responsiveness. Parallel data from 143 patients evaluated through immunohistopathological analyses could refine this concept pointing at baseline synovial $\mathrm{TNF} \alpha$ expression (but not IL1 $\beta$, IL6 or IL-18) as a significant predictor of response (change in 28-joint Disease Activity Score [DAS28] $\geq 1.2$ at week 16), accounting, together with baseline DAS28, for an increased explained variance of $17 \%$ (61). Integration of other histopathological markers (presence of sublining cellular aggregates) into a multivariable model including baseline DAS28, serum anti-citrullinated peptide antibodies and synovial TNF $\alpha$ improved the prediction of clinical response with an increased explained variance of $29 \%$ (62). A significant negative predictive role of synovial aggregates for anti-TNF treatment response in RA was instead reported in an independent study (86 patients) based on aggregate stratification according to the degree of lymphoid structural organization. Differences in the study population, endpoints or in the analytical method may account for this apparent discrepancy (63). Beyond specific results, these data, collectively, strengthen two relevant hypotheses (64):

1) the potential added value of synovial analysis in the development of prediction models for targeted therapies;

2) the possible value of dissecting and focusing on the specific cellular/molecular targets of a specific drug for the identification of predictive biomarkers.

Supporting this concept, recent studies based on synovial tissue analysis accord- 
ing to pathotype stratification (see Synovial pathotypes section) confirmed a positive association between anti-TNF clinical response and the myeloid phenotype, characteristically linked to $\mathrm{TNF} \alpha$-associated gene expression modules in RA (34).

\section{Predictors of response to rituximab}

In line with these perspectives, most of the studies that evaluated the synovium as a potential source of predictors of response to Rituximab have focused primarily on B cell biology (65-68). Thurlings et al. (66) analysed by serial biopsies (baseline, 4, 16 weeks) and immunohistochemistry the synovial tissue of 24 RA patients, investigating the dynamic relationship between CD22+ B cells, CD138+ plasma cells, follicular dendritic cells, macrophages, T cells and clinical response. Although none of the baseline characteristics of the synovium appeared relevant per se, changes between 4 and 16 weeks in CD138+ plasma cell infiltration demonstrated a significant predictive value for the size of DAS28 change at 24 weeks. Similarly, no differences between responders and non-responders were observed by Gutierrez-Roelens et al. (67) in baseline synovial distribution of CD20+ B cells, CD138+ plasma cells and other major populations, although a significantly higher expression of Ig k light chains was found in responders. Different results were instead obtained by Onno Teng et al. (68) who, in an independent study (25 patients), was able to observe a significantly lower distribution of different baseline $\mathrm{B}$ cell populations (CD20+ B cells, CD79a+ B cells and CD138+ plasma cells) in good responders. Patients displaying low expression of CD79a in the synovium had a significantly lower risk for a moderate or no response.

It is clear from this rapid overview on synovial predictors of response to Rituximab that available data are far from being conclusive. Although this might depend on the small samples size of the studied populations, other factors should be considered. These include a possible confounding effect derived from the high functional heterogeneity of B cells (not easy to be captured histologically) or the fact that Rituximab may act primarily outside of the joint, exerting only an indirect action in the synovium (69-71). Large multi-centre randomized clinical trials in anti-TNF inadequate responders focused on synovial biopsy are currently ongoing and are expected to provide more definite results (http://www.r4ra-nihr.whri.qmul.ac.uk).

\section{DRUG TAPERING AND WITHDRAWAL: DEVELOPMENT OF PROGNOSTIC BIOMARKERS OF REMISSION STABILITY}

Early diagnosis and treatment, better monitoring strategies and the availability of different biologic therapies have considerably improved the outcome of chronic inflammatory arthritis in recent years, with the achievement of stable remission in a sizable proportion of the cases. If, on the one hand, these progresses have changed patients' health and societal perspectives, on the other they have introduced new challenges. In particular, it remains undetermined in which patients achieving remission, treatments can be tapered or withdrawn with maintenance of health. This question, of fundamental relevance in clinical terms, has deep pathobiological roots. Indeed, differently from other diseases, such as cancers or infectious conditions in which the primary pathogenic elements can be tracked, the constructs remission, resolution and cure can be difficult to put into practice in RA or SpA due to lack of knowledge about basic disease processes. Thus, although several studies have contributed to refining the concept of clinical remission, the characterization of remission in pathobiological terms is still largely incomplete. In keeping with this goal, beside the growing number of studies that have investigated remission stability after drug tapering/suspension through clinical and imaging approaches (72-77), there is now increasing interest in addressing this issue also from the histopathological perspective.

In a recent exploratory study, Ramirez et 
al. (78) analysed by US-guided biopsy the histopathological features of power Doppler (PD) positive synovitis in 20 RA patients treated with synthetic or biologic disease-modifying anti-rheumatic drugs who achieved stable DAS28 remission with no tender and swollen joints. Progression to symptomatic, clinically active disease during a follow-up of 12 months was observed under treatment in 8 out of 20 patients and was associated with the degree of residual CD20+ B cell and mast cell infiltration. These data suggest a potential role of synovial histopathology for deeper stratification of PD positive clinical remission, thus in the presence of US-detectable sub-clinical inflammation. Another fundamental issue is, however, the analysis of the role of synovial histopathology in the stratification of PD negative clinical remission. In this context, recent data published by Alivernini et al. $(79,80)$ have offered important insights, suggesting the possibility of disease-related differences. In particular, as inferred by a cross-sectional analysis in patients under TNF inhibition, PD negative patients with PsA in clinical remission can display significantly higher degrees of residual inflammation (macrophages, $T$ cells and vascular structures) than PD negative patients with RA in remission, despite comparable clinical characteristics. Prospective studies performing treatment tapering or discontinuation, based on the combination of clinical, US and histological selection criteria are expected to define the prognostic significance of these observations.

\section{CONCLUSIONS}

Chronic inflammatory arthritides are characterized by a degree of phenotypic heterogeneity whose pathologic substrate remains largely undetermined. This is reflected in prognostic variability and variability in response to treatments. Currently available prediction tools remain unsatisfactory and do not allow to deal entirely with such complexity. Synovial tissue analysis has been an essential tool to improve our understanding of arthritis pathogenesis and for the development of new treatments. While developments in this direction should be still considered a priority, one of the next steps will be to define its applicability in real-life clinical practice.

Data presented in this review emphasize two main concepts:

1) the existence of a large amount of data that would actually support the possible exploitation of synovial pathology for diagnostic and prognostic purposes in different phases of disease progression;

2) the discrepancy between the amount of data generated so far and their poor exploitation by physicians and patients (81).

To explain in part this discrepancy there are some aspects of the approach to synovial tissue collection that should be considered. Firstly, synovial biopsy, although "mini", remains an invasive procedure that requires training, skills and facilities. Biomarkers eventually derived from synovial tissue need therefore to be characterized by high performance to be attractive. Secondly, current biopsy techniques do not guarantee uniform sampling efficiency across all joints and disease activity phases. Although large and small joints can be biopsied, the output is variable and can be influenced by several anatomic and disease-related factors (48). In the absence of highly trained operators, this may restrict applicability to a proportion of candidate subjects. Further technical developments in the biopsy procedure, the institution of international training programs and the validation of candidate biomarkers in prospective multicentre studies based on large populations are likely to promote translational development of the field.

\section{REFERENCES}

1. Shiboski CH, Shiboski SC, Seror R, et al. 2016 American College of Rheumatology/ European League Against Rheumatism classification criteria for primary Sjogren's syndrome: A consensus and data-driven methodology involving three international patient cohorts. Ann Rheum Dis. 2017; 76: 9-16.

2. Wilhelmus S, Alpers CE, Cook HT, et al. The Revisited Classification of GN in SLE at 10 
Years: Time to Re-Evaluate Histopathologic Lesions. J Am Soc Nephrol. 2015; 26: 2938-46.

3. Feakins RM, and British Society of G. Inflammatory bowel disease biopsies: updated British Society of Gastroenterology reporting guidelines. J Clin Pathol. 2013; 66: 1005-26.

4. Bresnihan B. Are synovial biopsies of diagnostic value? Arthritis Res Ther. 2003; 5: 271-8.

5. Manzo A, Paoletti S, Carulli M, Blades MC, Barone F, Yanni G, et al. Systematic microanatomical analysis of CXCL13 and CCL21 in situ production and progressive lymphoid organization in rheumatoid synovitis. Eur $\mathrm{J}$ Immunol. 2005; 35: 1347-59.

6. Corsiero E, Bombardieri M, Carlotti E, et al. Single cell cloning and recombinant monoclonal antibodies generation from RA synovial B cells reveal frequent targeting of citrullinated histones of NETs. Ann Rheum Dis. 2016; 75: 1866-75.

7. Manzo A, Vitolo B, Humby F, et al. Mature antigen-experienced $\mathrm{T}$ helper cells synthesize and secrete the $\mathrm{B}$ cell chemoattractant CXCL13 in the inflammatory environment of the rheumatoid joint. Arthritis Rheum. 2008; 58: 3377-87.

8. Rao DA, Gurish MF, Marshall JL, et al. Pathologically expanded peripheral $\mathrm{T}$ helper cell subset drives B cells in rheumatoid arthritis. Nature. 2017; 542: 110-4.

9. Bresnihan B, Pontifex E, Thurlings RM, et al. Synovial tissue sublining CD68 expression is a biomarker of therapeutic response in rheumatoid arthritis clinical trials: consistency across centers. J Rheumatol. 2009; 36: 1800-2.

10. Buch MH, Boyle DL, Rosengren S, et al. Mode of action of abatacept in rheumatoid arthritis patients having failed tumour necrosis factor blockade: a histological, gene expression and dynamic magnetic resonance imaging pilot study. Ann Rheum Dis. 2009; 68: 1220-7.

11. Polzer K, Baeten D, Soleiman A, et al. Tumour necrosis factor blockade increases lymphangiogenesis in murine and human arthritic joints. Ann Rheum Dis. 2008; 67: 1610-6.

12. Boyle DL, Soma K, Hodge J, et al. The JAK inhibitor tofacitinib suppresses synovial JAK1-STAT signalling in rheumatoid arthritis. Ann Rheum Dis. 2015; 74: 1311-6.

13. Orr C, Sousa E, Boyle DL et al. Synovial tissue research: a state-of-the art review. Nat Rev Rheumatol. 2017; 13: 463-75.

14. Mohr W, Beneke G, Mohing W. Proliferation of synovial lining cells and fibroblasts. Ann Rheum Dis. 1975; 34: 219-24.

15. De Bari C, Dell'Accio F, Tylzanowski P, Luyten FP. Multipotent mesenchymal stem cells from adult human synovial membrane. Arthritis Rheum. 2001; 44: 1928-42.
16. Kennedy A, Ng CT, Biniecka M, et al. Angiogenesis and blood vessel stability in inflammatory arthritis. Arthritis Rheum. 2010; 62: 711-21.

17. Canete JD, Santiago B, Cantaert T, et al. Ectopic lymphoid neogenesis in psoriatic arthritis. Ann Rheum Dis. 2007; 66: 720-6.

18. Qu Z, Garcia CH, O'Rourke LM, et al. Local proliferation of fibroblast-like synoviocytes contributes to synovial hyperplasia. Results of proliferating cell nuclear antigen/cyclin, cmyc, and nucleolar organizer region staining. Arthritis Rheum. 1994; 37: 212-20.

19. Ceponis A, Konttinen YT, Imai S, et al. Synovial lining, endothelial and inflammatory mononuclear cell proliferation in synovial membranes in psoriatic and reactive arthritis: a comparative quantitative morphometric study. Br J Rheumatol. 1998; 37: 170-8.

20. Ospelt C, Gay S, Klein K. Epigenetics in the pathogenesis of RA. Semin Immunopathol. 2017; 39: 409-19.

21. Dechanet J, Merville P, Durand I, et al. The ability of synoviocytes to support terminal differentiation of activated B cells may explain plasma cell accumulation in rheumatoid synovium. J Clin Invest. 1995; 95: 456-63.

22. Storch H, Zimmermann B, Resch B, et al. Activated human $B$ cells induce inflammatory fibroblasts with cartilage-destructive properties and become functionally suppressed in return. Ann Rheum Dis. 2016; 75: 924-32.

23. Manzo A, Bugatti S, Caporali R, et al. CCL21 expression pattern of human secondary lymphoid organ stroma is conserved in inflammatory lesions with lymphoid neogenesis. Am J Pathol. 2007; 171: 1549-62.

24. Krenn V, Schalhorn N, Greiner A, et al. Immunohistochemical analysis of proliferating and antigen-presenting cells in rheumatoid synovial tissue. Rheumatol Int. 1996; 15: 239-47.

25. Voswinkel J, Weisgerber K, Pfreundschuh M, Gause A. B lymphocyte involvement in ankylosing spondylitis: the heavy chain variable segment gene repertoire of B lymphocytes from germinal center-like foci in the synovial membrane indicates antigen selection. Arthritis Res. 2001; 3: 189-95.

26. Schroder AE, Greiner A, Seyfert C, Berek C. Differentiation of $\mathrm{B}$ cells in the nonlymphoid tissue of the synovial membrane of patients with rheumatoid arthritis. Proc Natl Acad Sci USA. 1996; 93: 221-5.

27. Humby F, Bombardieri M, Manzo A, et al. Ectopic lymphoid structures support ongoing production of class-switched autoantibodies in rheumatoid synovium. PLoS Med. 2009; 6: e1.

28. Bugatti S, Manzo A, Bombardieri M, et al. Synovial tissue heterogeneity and peripheral blood biomarkers. Curr Rheumatol Rep. 2011; 13: 440-8. 
29. Bugatti S, Manzo A, Vitolo B, et al. High expression levels of the B cell chemoattractant CXCL13 in rheumatoid synovium are a marker of severe disease. Rheumatology (Oxford). 2014; 53: 1886-95.

30. van der Pouw Kraan TC, van Gaalen FA, Huizinga TW, et al. Discovery of distinctive gene expression profiles in rheumatoid synovium using cDNA microarray technology: evidence for the existence of multiple pathways of tissue destruction and repair. Genes Immun. 2003; 4: 187-96.

31. van der Pouw Kraan TC, van Gaalen FA, Kasperkovitz PV, et al. Rheumatoid arthritis is a heterogeneous disease: evidence for differences in the activation of the STAT-1 pathway between rheumatoid tissues. Arthritis Rheum. 2003; 48: 2132-45.

32. Timmer TC, Baltus B, Vondenhoff M, et al. Inflammation and ectopic lymphoid structures in rheumatoid arthritis synovial tissues dissected by genomics technology: identification of the interleukin-7 signaling pathway in tissues with lymphoid neogenesis. Arthritis Rheum. 2007; 56: 2492-502.

33. Takemura S, Braun A, Crowson C, et al. Lymphoid neogenesis in rheumatoid synovitis. $\mathbf{J}$ Immunol. 2001; 167: 1072-80.

34. Dennis G Jr., Holweg CT, Kummerfeld SK, et al. Synovial phenotypes in rheumatoid arthritis correlate with response to biologic therapeutics. Arthritis Res Ther. 2014; 16: R90.

35. Baeten D, Demetter P, Cuvelier C, et al. Comparative study of the synovial histology in rheumatoid arthritis, spondyloarthropathy, and osteoarthritis: influence of disease duration and activity. Ann Rheum Dis. 2000; 59: 945-53.

36. Kraan MC, Reece RJ, Smeets TJ, et al. Comparison of synovial tissues from the knee joints and the small joints of rheumatoid arthritis patients: Implications for pathogenesis and evaluation of treatment. Arthritis Rheum. 2002; 46: 2034-8.

37. Koski JM, Helle M. Ultrasound guided synovial biopsy using portal and forceps. Ann Rheum Dis. 2005; 64: 926-9.

38. Saaibi DL, and Schumacher HR, Jr. Percutaneous needle biopsy and synovial histology. Baillieres Clin Rheumatol. 1996; 10: 535-54.

39. van de Sande MG, Thurlings RM, Boumans MJ, et al. Presence of lymphocyte aggregates in the synovium of patients with early arthritis in relationship to diagnosis and outcome: is it a constant feature over time? Ann Rheum Dis. 2011; 70: 700-3.

40. Kelly S, Humby F, Filer A, et al. Ultrasoundguided synovial biopsy: a safe, well-tolerated and reliable technique for obtaining highquality synovial tissue from both large and small joints in early arthritis patients. Ann Rheum Dis. 2015; 74: 611-7.

41. Kraan MC, Haringman JJ, Post WJ, et al. Immunohistological analysis of synovial tissue for differential diagnosis in early arthritis. Rheumatology (Oxford). 1999; 38: 1074-80.

42. Kane D, Veale DJ, FitzGerald O, Reece R. Survey of arthroscopy performed by rheumatologists. Rheumatology (Oxford). 2002; 41: 210-5.

43. Lazarou I, D’Agostino MA, Naredo E, et al. Ultrasound-guided synovial biopsy: a systematic review according to the OMERACT filter and recommendations for minimal reporting standards in clinical studies. Rheumatology (Oxford). 2015; 54: 1867-75.

44. Youssef PP, Kraan M, Breedveld F, et al. Quantitative microscopic analysis of inflammation in rheumatoid arthritis synovial membrane samples selected at arthroscopy compared with samples obtained blindly by needle biopsy. Arthritis Rheum. 1998; 41: 663-9.

45. Scire CA, Epis O, Codullo V, et al. Immunohistological assessment of the synovial tissue in small joints in rheumatoid arthritis: validation of a minimally invasive ultrasound-guided synovial biopsy procedure. Arthritis Res Ther. 2007; 9: R101.

46. Dolhain RJ, Ter Haar NT, De Kuiper R, et al. Distribution of $\mathrm{T}$ cells and signs of T-cell activation in the rheumatoid joint: implications for semiquantitative comparative histology. $\mathrm{Br}$ J Rheumatol. 1998; 37: 324-30.

47. Boyle DL, Rosengren S, Bugbee W, et al. Quantitative biomarker analysis of synovial gene expression by real-time PCR. Arthritis Res Ther. 2003; 5: R352-60.

48. Humby F, Kelly S, Hands R, et al. Use of ultrasound-guided small joint biopsy to evaluate the histopathologic response to rheumatoid arthritis therapy: recommendations for application to clinical trials. Arthritis Rheum. 2015; 67: 2601-10.

49. Zink A, Albrecht K. Rheumatoid arthritis: the benefits of early treatment after decades. Nat Rev Rheumatol. 2017; 13: 458-9.

50. Haroon M, Gallagher P, FitzGerald O. Diagnostic delay of more than 6 months contributes to poor radiographic and functional outcome in psoriatic arthritis. Ann Rheum Dis. 2015; 74: 1045-50.

51. Veale D, Yanni G, Rogers S, et al. Reduced synovial membrane macrophage numbers, ELAM-1 expression, and lining layer hyperplasia in psoriatic arthritis as compared with rheumatoid arthritis. Arthritis Rheum. 1993; 36: 893-900.

52. Kruithof E, Baeten D, De Rycke L, et al. Synovial histopathology of psoriatic arthritis, both oligo- and polyarticular, resembles spondyloarthropathy more than it does rheumatoid 
arthritis. Arthritis Res Ther. 2005; 7: R569-80.

53. Fearon U, Griosios K, Fraser A, et al. Angiopoietins, growth factors, and vascular morphology in early arthritis. J Rheumatol. 2003; 30: $260-8$.

54. van de Sande MG, de Launay D, de Hair MJ, et al. Local synovial engagement of angiogenic TIE-2 is associated with the development of persistent erosive rheumatoid arthritis in patients with early arthritis. Arthritis Rheum. 2013; 65: 3073-83.

55. Baeten D, Moller HJ, Delanghe J, et al. Association of CD163+ macrophages and local production of soluble CD163 with decreased lymphocyte activation in spondylarthropathy synovitis. Arthritis Rheum. 2004; 50: 1611-23.

56. Baeten D, Steenbakkers PG, Rijnders AM, et al. Detection of major histocompatibility complex/human cartilage gp-39 complexes in rheumatoid arthritis synovitis as a specific and independent histologic marker. Arthritis Rheum. 2004; 50: 444-51.

57. Yeremenko N, Noordenbos T, Cantaert T, et al. Disease-specific and inflammation-independent stromal alterations in spondylarthritis synovitis. Arthritis Rheum. 2013; 65: 174-85.

58. Baeten D, Kruithof E, De Rycke L, et al. Diagnostic classification of spondylarthropathy and rheumatoid arthritis by synovial histopathology: a prospective study in 154 consecutive patients. Arthritis Rheum. 2004; 50: 2931-41.

59. Bergman GJ, Hochberg MC, Boers M, et al. Indirect comparison of tocilizumab and other biologic agents in patients with rheumatoid arthritis and inadequate response to diseasemodifying antirheumatic drugs. Semin Arthritis Rheum. 2010; 39: 425-41.

60. van der Pouw Kraan TC, Wijbrandts CA, van Baarsen LG, et al. Responsiveness to anti-tumour necrosis factor alpha therapy is related to pre-treatment tissue inflammation levels in rheumatoid arthritis patients. Ann Rheum Dis. 2008; 67: 563-6.

61. Wijbrandts CA, Dijkgraaf MG, Kraan MC, et al. The clinical response to infliximab in rheumatoid arthritis is in part dependent on pretreatment tumour necrosis factor alpha expression in the synovium. Ann Rheum Dis. 2008; 67: 1139-44.

62. Klaasen R, Thurlings RM, Wijbrandts CA, et al. The relationship between synovial lymphocyte aggregates and the clinical response to infliximab in rheumatoid arthritis: a prospective study. Arthritis Rheum. 2009; 60: 3217-24.

63. Canete JD, Celis R, Moll C, et al. Clinical significance of synovial lymphoid neogenesis and its reversal after anti-tumour necrosis factor alpha therapy in rheumatoid arthritis. Ann Rheum Dis. 2009; 68: 751-6.
64. Ulfgren AK, Andersson U, Engstrom M, et al. Systemic anti-tumor necrosis factor alpha therapy in rheumatoid arthritis down-regulates synovial tumor necrosis factor alpha synthesis. Arthritis Rheum. 2000; 43: 2391-6.

65. Kavanaugh A, Rosengren S, Lee SJ, et al. Assessment of rituximab's immunomodulatory synovial effects (ARISE trial). 1: clinical and synovial biomarker results. Ann Rheum Dis. 2008; 67: 402-8.

66. Thurlings RM, Vos K, Wijbrandts CA, Zwinderman AH, et al. Synovial tissue response to rituximab: mechanism of action and identification of biomarkers of response. Ann Rheum Dis. 2008; 67: 917-25.

67. Gutierrez-Roelens I, Galant C, Theate I, et al. Rituximab treatment induces the expression of genes involved in healing processes in the rheumatoid arthritis synovium. Arthritis Rheum. 2011; 63: 1246-54.

68. Teng YK, Levarht EW, Hashemi M, et al. Immunohistochemical analysis as a means to predict responsiveness to rituximab treatment. Arthritis Rheum. 2007; 56: 3909-18.

69. Bugatti S, Vitolo B, Caporali R, et al. B cells in rheumatoid arthritis: from pathogenic players to disease biomarkers. Biomed Res Int. 2014; 2014: 681678.

70. Manzo A, Caporali R, Vitolo B, et al. Subclinical remodelling of draining lymph node structure in early and established rheumatoid arthritis assessed by power Doppler ultrasonography. Rheumatology (Oxford). 2011; 50: 1395-400.

71. Bugatti S, Manzo A, Caporali R, Montecucco C. Inflammatory lesions in the bone marrow of rheumatoid arthritis patients: a morphological perspective. Arthritis Res Ther. 2012; 14: 229.

72. Klarenbeek NB, van der Kooij SM, GulerYuksel M, et al. Discontinuing treatment in patients with rheumatoid arthritis in sustained clinical remission: exploratory analyses from the BeSt study. Ann Rheum Dis. 2011; 70: 315-9.

73. Rech J, Hueber AJ, Finzel S, et al. Prediction of disease relapses by multibiomarker disease activity and autoantibody status in patients with rheumatoid arthritis on tapering DMARD treatment. Ann Rheum Dis. 2016; 75: 1637-44.

74. Araujo EG, Finzel S, Englbrecht M, et al. High incidence of disease recurrence after discontinuation of disease-modifying antirheumatic drug treatment in patients with psoriatic arthritis in remission. Ann Rheum Dis. 2015; 74: 655-60.

75. Tanaka Y, Hirata S, Kubo S, et al. Discontinuation of adalimumab after achieving remission in patients with established rheumatoid arthritis: 1-year outcome of the HONOR study. Ann Rheum Dis. 2015; 74: 389-95. 
76. Figueiredo CP, Bang H, Cobra JF, et al. Antimodified protein antibody response pattern influences the risk for disease relapse in patients with rheumatoid arthritis tapering disease modifying antirheumatic drugs. Ann Rheum Dis. 2017; 76: 399-407.

77. Saleem B, Keen H, Goeb V, et al. Patients with RA in remission on TNF blockers: when and in whom can TNF blocker therapy be stopped? Ann Rheum Dis. 2010; 69: 1636-42.

78. Ramirez J, Celis R, Usategui A, et al. Immunopathologic characterization of ultrasounddefined synovitis in rheumatoid arthritis patients in clinical remission. Arthritis Res Ther. 2016; $18: 74$.
79. Alivernini S, Peluso G, Fedele AL, et al. Tapering and discontinuation of TNF-alpha blockers without disease relapse using ultrasonography as a tool to identify patients with rheumatoid arthritis in clinical and histological remission. Arthritis Res Ther. 2016; 18: 39.

80. Alivernini S, Tolusso B, Petricca L, et al. Synovial features of patients with rheumatoid arthritis and psoriatic arthritis in clinical and ultrasound remission differ under anti-TNF therapy: a clue to interpret different chances of relapse after clinical remission? Ann Rheum Dis. 2017; 76: 1228-36.

81. Butler D. Translational research: crossing the valley of death. Nature. 2008; 453: 840-2. 